\title{
P01-93
}

\section{BENEFICIAL EFFECTS OF MEMANTINE IN EVERY DAY MEDICAL PRACTICE: RESULTS FROM A LARGE GREEK OBSERVATIONAL STUDY}

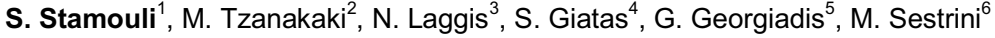 \\ ${ }^{1}$ Department of Psychiatry, Eginition University Hospital, Athens, ${ }^{2}$ Center for Mental Health, General Hospital of Chania, Chania, \\ ${ }^{3}$ Department of Neurology, 'Elpis' General Hospital, ${ }^{4}$ Department of Neurology, 251 Military Hospital, Athens, ${ }^{5}$ Department of \\ Neurology, Ippokrateion Hospital of Thessaloniki, Thessaloniki, ${ }^{6}$ Department of Psychiatry, Sismanogleion Hospital of Athens, \\ Athens, Greece
}

Memantine, NMDA-R antagonist, is approved for the treatment of moderate-to-severe Alzheimer's disease (MMSE< 20) (AD). The purpose of this study was to evaluate the efficacy and safety of memantine when used in routine clinical practice.

The 6-months, observational, open-label, multicentre study in 202 specialist centers in Greece evaluated the efficacy of memantine using the MMSE and Instrumental Activities of Daily Living (IADL) scale at baseline, 3 and 6 months. Safety was evaluated by spontaneously reported adverse events (AEs). Statistical efficacy analyses were performed in the Intent-To-Treat (ITT) (at least one post-baseline evaluation) and Per Protocol (PP) datasets (evaluations at both 3 and 6 months).

The study included 2570 AD patients (age: $74.8 \pm 6.8,54.6 \%$ women, baseline MMSE score: $18.0 \pm 5.0$ ). $34.2 \%$ had received previous treatment with acetylocholinesterase inhibitors (AChEls), while for $65.8 \%$ memantine was the first treatment option. At baseline $91.5 \%$ were prescribed memantine as monotherapy, the remainder also received AChEls. During the 6 months of the study, $80.9 \%$ continued memantine monotherapy. MMSE score was significantly improved from baseline at $3(17.9 \pm 5.1 \mathrm{vs} 19.2 \pm 5.0, p<0.001$, repeated measures analysis of variance Hotelling's test, ITT) and 6 months (17.9 \pm 5.1 vs $19.7 \pm 5.1)$. At 6 months, $67 \%$ of the ITT population had improved their MMSE score and $18.6 \%$ had no change. 19 patients $(0.7 \%)$ terminated the therapy prematurely due to AEs. AEs were reported in $182(7.1 \%)$ patients: the most common was dizziness $(1.45 \%) .8(0.3 \%)$ of the AEs were severe. These results in naturalistic settings support the excellent efficacy and tolerability profile of memantine. 